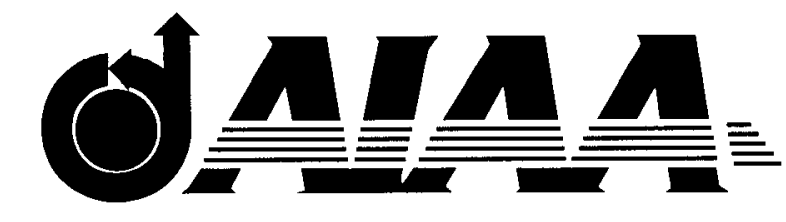

\title{
AIAA 98-2611 \\ Digital PIV Measurements of Acoustic Particle Displacements in a Normal Incidence Impedance Tube
}

William M. Humphreys, Jr.

Scott M. Bartram

Tony L. Parrott

Michael G. Jones

NASA Langley Research Center Hampton, VA 23681-0001

\section{0th AIAA Advanced Measurement and Ground Testing Technology Conference June 15-18, 1998 / Albuquerque, NM}


AIAA-98-2611

\title{
DIGITAL PIV MEASUREMENTS OF ACOUSTIC PARTICLE DISPLACEMENTS IN A NORMAL INCIDENCE IMPEDANCE TUBE
}

\author{
William M. Humphreys, Jr." \\ Scott M. Bartram ${ }^{\dagger}$ \\ Tony L. Parrott ${ }^{\ddagger}$ \\ Michael G. Jones' \\ Fluid Mechanics and Acoustics Division \\ NASA Langley Research Center \\ Hampton, Virginia 23681-0001
}

\begin{abstract}
Acoustic particle displacements and velocities inside a normal incidence impedance tube have been successfully measured for a variety of pure tone sound fields using Digital Particle Image Velocimetry (DPIV). The DPIV system utilized two 600-mj Nd:YAG lasers to generate a double-pulsed light sheet synchronized with the sound field and used to illuminate a portion of the oscillatory flow inside the tube. A high resolution (1320 x 1035 pixel), 8-bit camera was used to capture double-exposed images of $2.7-\mu \mathrm{m}$ hollow silicon dioxide tracer particles inside the tube. Classical spatial autocorrelation analysis techniques were used to ascertain the acoustic particle displacements and associated velocities for various sound field intensities and frequencies. The results show that particle displacements spanning a range of $1-60 \mu \mathrm{m}$ can be measured for incident sound pressure levels of $100-130 \mathrm{~dB}$ and for frequencies spanning $500-1000 \mathrm{~Hz}$. The ability to resolve $1 \mu \mathrm{m}$ particle displacements at sound pressure levels in the $100 \mathrm{~dB}$ range allows the use of DPIV systems for measurement of sound fields at much lower sound pressure levels than had been previously possible. Representative impedance tube
\end{abstract}

*Research Scientist, Measurement Science and Technology

Branch, Senior Member AIAA.

${ }^{\dagger}$ Engineering Technician, Measurement Science and

Technology Branch.

${ }^{\ddagger}$ Senior Research Scientist, Structural Acoustics Branch.

'Research Scientist, Structural Acoustics Branch.

Copyright $(1998$ by the American Institute of Aeronautics and Astronautics, Inc. No copyright is asserted in the United States under Title 17, U.S. Code. The U.S. Government has a royaltyfree license to exercise all rights under the copyright claimed herein for government purposes. All other rights are reserved by the copyright owner. data as well as an uncertainty analysis for the measurements are presented.

\section{NOMENCLATURE}

$c_{0}$

$C_{c}$

Speed of sound, $\mathrm{m} / \mathrm{sec}$

Cunningham correction factor for

molecular slip

$d_{c} \quad$ Physical distance between pixels in camera, $\mathrm{m}$

Measured tick mark spacing, $\mathrm{m}$

Particle diameter, $\mathrm{m}$

DPIV particle displacement vector, $\mathrm{m}$

Spatial autocorrelation displacement vector, $\mathrm{m}$

$\overrightarrow{\mathrm{d}}_{s}$ $e^{-i \omega t}$

$k$

$L$

$M$

$N_{p}$

$\hat{n}$

$\mathrm{p}$

$\overrightarrow{\mathrm{u}}$

$\overrightarrow{\mathrm{V}}$

$\vec{x}$

$Z$

$\Delta \tau$

$\bar{\eta}$

Calcite window spatial image shift, $\mathrm{m}$

Time convention

Wavenumber, $\left(=\omega / \mathrm{c}_{\mathrm{o}}\right), \mathrm{m}^{-1}$

Length of normal incidence tube, $m$ Magnification

Number of pixels between grid tick marks

Unit vector normal to surface

Complex pressure, Pascals

Complex acoustic particle velocity, $\mathrm{m} / \mathrm{sec}$

DPIV particle velocity vector, $\mathrm{m} / \mathrm{sec}$

Position vector, meters

Complex acoustic impedance

Laser pulse separation, sec

Average fluid viscosity, $\mathrm{N}-\mathrm{sec} / \mathrm{m}^{2}$

Gas density, $\mathrm{kg} / \mathrm{m}^{3}$ 


$\begin{array}{ll}\sigma_{x}^{2} & \text { Variance of variable } \mathrm{x} \\ \tau_{p} & \text { Particle dynamic relaxation time, sec } \\ \mu_{x} & \text { Mean of variable } \mathrm{x} \\ \mu_{p} & \text { Particle tracking fidelity } \\ \omega & \text { Radian frequency, }(=2 \pi \mathrm{f}), \mathrm{rad} / \mathrm{sec} \\ \xi & \text { Acoustic particle displacement, } \mathrm{m}\end{array}$

\section{INTRODUCTION}

There exists a continual need for the reduction of generated noise by commercial aircraft turbofan engines. Both the mandated Federal Aviation Administration noise reduction goals and the NASA Advanced Subsonic Technology (AST) level 1 goals detail a greater than $10 \mathrm{~dB}$ reduction in effective perceived noise (EPN) by the year 2000, relative to levels measured in 1992. This reduction in turbofan noise is complicated by the large number of potential noise sources present in modern engines (e.g., noise generated by inflow disturbances, fan and turbine exhaust, etc.). A common method for turbofan engine noise reduction involves the use of passive liners along the interior walls of the engine nacelle. Such liners have been studied extensively over the last three decades, and have accounted for approximately $6 \mathrm{~dB}$ of EPN reductions. However, the current mandates require another $3 \mathrm{~dB}$ of noise reduction by the year 2000 , with greater attenuation in the $0.4-4.0 \mathrm{kHz}$ portion of the spectrum. The search for appropriate passive nacelle liner structures to provide this bandwidth improvement as well as better efficiency involves interdisciplinary research. This includes computational modeling and development of new types of instrumentation which can measure the effects of flow and sound pressure levels on the performance of the liner structure.

One of the parameters which is used to characterize nacelle liner performance is the normal incidence acoustic impedance spectrum, defined as the ratio of the Fourier transform of the acoustic pressure to that of the acoustic particle velocity measured normal to a given surface. ${ }^{1}$ This is represented mathematically as

$$
Z_{\hat{n}}(\vec{x}, \omega)=\frac{p(\vec{x}, \omega)}{\overrightarrow{\mathrm{u}}(\vec{x}, \omega) \cdot \hat{n}}
$$

where $Z$ and $p$ denote complex scalars and $\overrightarrow{\mathrm{u}}$ denotes a complex vector. Knowledge of the acoustic impedance is important because it allows the calculation of acoustic intensity in a given direction, provided the RMS acoustic pressure is known. The impedance also provides boundary conditions for analytical and numerical duct propagation models for computation of noise suppression. A number of classical methods for indirect measurement of the acoustic impedance spectrum have been described in the literature by Jones and Stiede. ${ }^{2}$ However, calculation of the impedance by separate direct measure of the pressure and particle velocity fields has traditionally been difficult, given the relatively small amplitude motions of the fluid particles in response to the sound field. Optical diagnostic techniques have been applied by a number of researchers to attempt direct measure of acoustic particle velocity fields. The most notable work is that of Sharpe and Greated, who developed photon correlation techniques from which complex acoustic impedance could be measured. ${ }^{3-4}$ More recently, Particle Image Velocimetry (PIV) techniques have been applied to sound fields with the work of Sharpe, et.al., ${ }^{5}$ Arroyo and Greated, ${ }^{6}$ and Hann and Greated. ${ }^{7}$ This work focused on measurement of acoustic particle displacement and flow velocity in the presence of acoustic streaming generated by high-intensity $(>130 \mathrm{~dB})$ sound fields. ${ }^{8}$

While the measurement of particle displacement and velocity in a nonlinear acoustic medium is useful for high intensity sound applications where streaming is desired, the authors decided to perform a feasibility study to determine whether it is practical to attempt similar optical diagnostics in a lower intensity sound field. Acoustic streaming is not present in this case, and acoustic particle displacements are an order of magnitude smaller, which represents a significant measurement challenge. Nevertheless, such measurements would allow the acoustic impedance spectrum given in equation (1) to be measured directly over a wider range of sound intensities. To obtain acoustic particle displacement data under controlled conditions, a series of experiments were performed in a sealed rectangular chamber known as an impedance tube. Such tubes are routinely used to characterize the impedance of locally reacting structures found in engine nacelles. For the purposes of this investigation, a normal incidence tube (NIT) configuration was chosen with an acoustic source, a short waveguide, and a highly reflective termination combined in a linear arrangement, as shown in Figure 1. The reflective termination creates a strong standing wave pattern along the length of the tube. For a single pure tone introduced into the tube, the acoustic particle velocity and displacement as a function of axial location in the tube are represented mathematically by 


$$
\begin{gathered}
u(x)=\frac{-i}{\rho c_{o}}\left[|P(L)| e^{i k L} \operatorname{Sin} k(L-x)\right] \\
\xi(x)=\frac{i}{\omega} u(x)
\end{gathered}
$$

with the RMS pressure measured at the surface of the endplate given by

$$
|P(L)|=(20 \mu \text { Pascals }) 10^{\text {SPLi20 }}
$$

where $\rho$ is the gas density in the tube, $c_{o}$ is the speed of sound, $\omega$ is the radial frequency, $k$ is the wavenumber, and $S P L$ is the sound pressure level in $\mathrm{dB}$ measured at the termination, referenced to $20 \mu$ Pascals. Operated without a bias flow, equation (2) describes a zero-mean oscillatory flow of air within the tube in response to the sound field.

The basis of the study consists of applying PIV techniques to obtain images of entrained particles inside the NIT, from which the acoustic particle field can be measured on a global scale for comparison with predicted values obtained using equations (2) and (3). PIV is now widely accepted in the fluid mechanics research community to acquire instantaneous, twodimensional planar velocity data nonintrusively in a variety of flows, and has been used by the authors in numerous applications. ${ }^{9}$ A high resolution video camera was used in lieu of more traditional wet-process photography for this study both to expedite the turnaround time required to obtain particle displacement and velocity data from the raw captured images and to allow the progress of the experiment to be easily monitored. Incorporation of a digital camera in the data acquisition system allowed the use of advanced image processing techniques such as adaptive background subtraction. A digital camera also allowed the quality and density of particle images to be ascertained immediately upon acquisition, a feature which proved to be of great importance to the success of the measurements.

A description of the NIT and Digital PIV (DPIV) system used to perform the study is presented here. Several examples of acoustic particle displacement and velocity data obtained in the NIT are presented, as well as an uncertainty analysis for these measurements.

\section{EXPERIMENTAL SETUP}

Normal Incidence Impedance Tube: The NIT, pictured in Figure 2, consisted of an aluminum waveguide with a 50.8 by $50.8 \mathrm{~mm}$ interior cross section dimension and a length of 0.58 meters. The exit of the waveguide contained a docking port to which a termination was mounted normal to the sound field. Ahead of the waveguide exit were a series of high quality windows, with the side windows measuring 50.8 by $50.8 \mathrm{~mm}$ and the top window measuring 63.5 by $15.9 \mathrm{~mm}$. A rotating drum assembly containing two flush-mounted microphones used to obtain indirect acoustic impedance measurements was located approximately 0.27 meters ahead of the termination in the top of the waveguide. An additional flush-mounted microphone was located next to the top window immediately in front of the termination. This microphone was used to both synchronize the DPIV laser system to the acoustic pressure wave and to monitor the sound pressure levels inside the waveguide. A 16-ohm speaker mounted at the entrance of the waveguide was driven by an arbitrary waveform generator connected to an 800 -Watt power amplifier. The power amplifier allowed frequencies spanning $500-1000 \mathrm{~Hz}$ at sound pressure levels of $90-135 \mathrm{~dB}$ to be generated.

DPIV Optical System: The DPIV optical system main components included two 600-mj Nd:YAG lasers for flow field illumination and a high-resolution digital camera for image capture. The two laser beams were aligned to the same optical axis using a half-wave retardation plate and a thin-film polarizing beamsplitter, shown in the schematic of Figure 3 . The combined beams were then fanned out into a thin light sheet using a $75-\mathrm{mm}$ concave cylindrical lens coupled with a 1000-mm plano-convex lens used to re-collimate the horizontal axis and focus the vertical axis near the top window of the NIT. This optical configuration produced a focused light sheet which was approximately an order of magnitude wider $(50-60 \mathrm{~mm})$ than the field of view of the digital camera, allowing several regions of the particle field inside the NIT to be surveyed by translating the camera and/or the NIT parallel to the light sheet without requiring realignment of the laser optics.

A major objective of this study was to determine the lower threshold of detection of acoustic particle displacement in the presence of a pure tone sound field. Therefore, the design of the digital camera lens system was considered critical if particle motions in the micrometer range were to be resolved. The final design of the camera system with receiver lens is shown in the schematic of Figure 4. A custom manufactured $500-\mathrm{mm}, \mathrm{f} / 2.5$ lens incorporating a one-to-one conjugate and designed for a wavelength of $532 \mathrm{~nm}$ 
(matching the output wavelength of the lasers) was utilized. This lens was capable of resolving two point sources located $20 \mu \mathrm{m}$ apart at $\mathrm{f} / 2.5$ with only $0.005 \%$ distortion introduced. The lens (which weighed approximately $9 \mathrm{~kg}$ ) was mounted to a single-axis, $10-\mu \mathrm{m}$ resolution translation stage, allowing it to be moved normal to the light sheet for focus adjustment. A magnification of approximately 1.1 was utilized, producing a field of view of 7.7 by $6.0 \mathrm{~mm}$. A $2-\mathrm{mm}$ thick calcite window was mounted between the lens and digital camera to resolve image displacement directionality while extending the dynamic range of measurement. The polarization of each laser was controlled such that the two individual light sheets were polarized perpendicularly. The calcite introduced a spatial shift in the image field for only one polarization, thereby introducing a bias in the acquired doubleexposed particle image field. This bias was subtracted during image analysis to allow the direction of particle displacements to be ascertained. ${ }^{10-11}$

The high resolution camera used for image acquisition contained a 1320 x 1035-pixel sensor connected to an 8-bit digitizer. The camera was connected to a PCI-based frame buffer via an RS-422 interface, and was operated in single frame mode using a mechanical shutter set for a 0.125 -second exposure. Typically, the Nd:YAG lasers were operated at a repetition rate of $10 \mathrm{~Hz}$ with a pulse separation of several hundred microseconds, assuring that each acquired image would contain one double-pulse laser sequence without requiring additional laser/camera synchronization circuitry.

Laser / Sound Field Synchronization: Since the motion of the air in the NIT is oscillatory, the firing of the laser system needed to be phase-locked with the pressure wave in the waveguide in the vicinity of the measurement region. Proper synchronization was critical if correct particle displacements as a function of sound pressure level and frequency were to be obtained. The reason for this can be seen in Figure 5. The inter-laser pulse separation must be set equal to one half of the acoustic wave period to record the maximum acoustic particle displacement for a particular sound pressure level and frequency, with the first laser pulse closely coinciding with the peak of the wave. Any deviation from this arrangement will result in recording an apparent particle displacement different from the true displacement. For instance, if the lasers are pulsed with a time separation equal to one full acoustic period, no apparent displacements will be recorded, a fluid particle having moved through its maximum displacement and back to its starting position by the time the second laser pulse occurs. Therefore, the laser system was synchronized with the acoustic pressure wave within the waveguide with the help of a microphone mounted near the measurement location, but far enough outside of the light sheet to avoid interference. The output of the microphone, which recorded the time history of the pressure field inside the NIT, was used to trigger the lasers at one particular phase angle of the wave. The trigger phase angle could be adjusted to match various frequencies introduced into the waveguide.

Particle Seeding: A particle field was injected into the waveguide to visualize the oscillatory air motion within the NIT and provide a mechanism for capturing this motion on the digital camera. A critical requirement was to use particles which would follow the motions of the fluid while remaining suspended long enough to complete a data acquisition cycle. Based on these requirements, $2.7-\mu \mathrm{m}$ hollow silicon dioxide spheres having an extremely low bulk density of $4.5 \times 10^{-4} \mathrm{~kg} / \mathrm{m}^{3}$ were chosen as the seed material. These particles also provided excellent preservation of the scattered laser light polarization. The requirement that the particles have low density was important given the fact that there was no flow inside the NIT - only an oscillatory motion generated by the sound field. Thus, particles with high specific gravity would settle out of the tube too quickly, limiting the amount of data which could be obtained. Also, a high settling rate would show up in the captured particle field images as an unwanted particle displacement bias, which was unacceptable. The silicon dioxide particles reduced this bias to a minimum. A detailed analysis of the ability of these particles to accurately follow the acoustic pressure wave will be given subsequently.

System Calibration: Calibration of the DPIV system consisted of accurately measuring four key parameters, namely the sensitivity of the trigger microphone in the waveguide, the laser pulse separation, the magnification of the receiver system, and the amount of induced image shift generated by the calcite window. The trigger microphone, which also doubled as a sound pressure level monitor, was calibrated with a sound level calibrator and FFT analyzer. After mounting the microphone in the NIT, the acoustic driver was turned on and the sound pressure level and frequency adjusted at the arbitrary function generator to give the desired values. The laser pulse separation was measured for each image acquisition by collecting a portion of each laser beam onto a photodetector connected to a high speed digital oscilloscope. This oscilloscope was also 
used to monitor both the output of the arbitrary function generator and the trigger microphone to verify proper synchronization of the lasers with the sound field.

The camera system magnification, $M$, was computed by capturing images of a precision chrome on glass grid situated in the plane of the light sheet ${ }^{12}$, using the relation

$$
M=\frac{\left(N_{p}\right)\left(d_{c}\right)}{d_{g}}
$$

where $d_{g}$ is the measured grid tick mark spacing, $N_{p}$ is the number of pixels between adjacent tick marks on the image of the grid (averaged over the entire field of view), and $d_{c}$ is the physical distance between adjacent pixels in the camera. Measurements of $M$ were taken both horizontally and vertically to check for consistency in the magnification readings. The two values were similar since the camera employed a detector consisting of square $6.8-\mu \mathrm{m}$ pixels with $100 \%$ fill between pixels.

The calibration of the calcite window image shifter required special care since electro-optical image shifters of this type are susceptible to non-uniformity in the induced spatial shift, caused by small variations in light sheet polarization angle. Thus, the calibration was achieved by setting both lasers to fire simultaneously (i.e., forcing the pulse separation between lasers to zero) and acquiring images of the illuminated seed within the NIT. In effect, the only image displacements recorded by the camera were due to the calcite window (the effect of distortions caused by the NIT windows having been assumed negligible), with the oscillatory movement of the particles having been frozen by the simultaneous laser firing. Processing these calibration images in the same manner as actual data images allows the variability of the image shift across the field of view of the camera to be easily ascertained and corrected.

Data Acquisition: For a typical acquisition run, an eye dropper was filled with silicon dioxide particles which were then injected into the NIT through one of the two mounting holes used for impedance measurements near the center of the waveguide. (Due to their extremely low density, the particles could be drawn up into the eye dropper like a fluid.) After injecting the seed into the NIT, a $6.35-\mathrm{mm}$ tube mounted through a stopper was fitted into the microphone hole. A rubber bulb (ear syringe) on the other end of the tube produced a puff of air when squeezed which dispersed the particles in the NIT.
Two or three puffs was enough agitation to fill the NIT with suspended particles. When this procedure was performed, the initial particle density was high enough that the acquisition camera would typically become saturated briefly. As the turbulent motion caused by the puff of air subsided, the particles began to settle out of the NIT very slowly due to gravity. This process was monitored until individual particles could be seen clearly. At this point 20 double-exposed images of the illuminated particle field were captured and stored on disk for future analysis.

Data Analysis: A custom automated analysis system built around a PC platform and incorporating an Intel i860-based accelerator board was developed for this study. ${ }^{13}$ The system was capable of generating a minimum of 5 image displacement vector measurements per second, and completed a typical $1320 \times 1035$ pixel image in 1 minute using $128 \times 128$-pixel interrogation regions spatially oversampled by $50 \%$ in both the horizontal and vertical directions. The average particle image displacement vector, $\overrightarrow{\mathrm{d}}$, occurring in each interrogation region image was obtained using classical spatial autocorrelation analysis, similar to that described by Adrian. ${ }^{14}$ Using this information, the average acoustic particle displacement, $\overrightarrow{\mathrm{D}}$, and velocity, $\overrightarrow{\mathrm{V}}$, associated with the image were obtained from

$$
\overrightarrow{\mathrm{D}}=\frac{\overrightarrow{\mathrm{d}}-\overrightarrow{\mathrm{d}}_{s}}{M}, \quad \overrightarrow{\mathrm{V}}=\frac{\overrightarrow{\mathrm{D}}}{\Delta \tau}
$$

where $\overrightarrow{\mathrm{d}}_{s}$ is the measured calcite window image shift at the interrogation region position, and $\Delta \tau$ is the laser pulse separation.

For each frequency and sound pressure level tested, a series of 20 captured images were analyzed. Analysis of each image generated a mean velocity field containing 285 vectors, yielding a total of 5700 vectors for the entire ensemble of 20 images. To obtain a highly accurate mean particle displacement over the entire field of view of the acquisition camera (approximately $42 \mathrm{~mm}^{2}$ ), these 5700 vectors were averaged to give one acoustic particle displacement and hence velocity for each test condition. This procedure yielded a statistically accurate measure of the acoustic particle displacement for the test condition averaged over the field of view of the camera. The authors decided to perform this averaging to reduce the standard error of the mean displacement measure, acknowledging that an assumption of acoustic particle 
displacement uniformity over the field of the view of the measurement is required. However, given the small field of view utilized for the measurements and the much larger acoustic wavelengths employed in the study, this assumption was reasonable.

Data Validation: Custom software was written to apply three types of automated data validation to the resultant velocity fields to ensure removal of spurious vectors. ${ }^{15}$ First, a bandpass filter was applied to the individual horizontal and vertical components of each vector in the velocity field. This operation verified that the components were within a specified range based on a priori knowledge of the expected values for the vectors. Second, a local median validator was applied to the individual vectors in the velocity field. This validator compared each vector (the test vector) with its nearest eight neighbors based on local median and variance levels. If the difference between the local median and the test vector was more than three standard deviations, the vector was considered invalid and removed. Finally, a $3 \times 3$ local mean filter was applied to the velocity field to provide both an interpolation function to fill in gaps resulting from the previous two validation procedures and to partially smooth the velocity field. Using these three procedures, validation rates exceeding $99 \%$ were achieved. In practice, very few vectors were removed during the validation process, in part due to the uniformity and relative high density ( $>5$ particle $/ \mathrm{mm}^{2}$ ) of seed particles across the field of view of the camera. This is illustrated in the example image of Figure 6.

\section{EXPERIMENTAL RESULTS}

Three sets of representative data obtained in the NIT during this study are shown. The first illustrates the apparent change in measured particle displacement as a function of synchronization phase between the acoustic pressure wave and the laser system. The second shows the dependence of acoustic particle displacement on sound pressure level for a pure $500 \mathrm{~Hz}$ tone. The final set of data shows the results of a centerline survey in the NIT from the termination to the opposite end of the viewing window, spanning a distance of approximately $50 \mathrm{~mm}$, using a pure $1000 \mathrm{~Hz}$ tone.

Synchronization Phase Variation: Data was taken at sixteen different phases for a $118-\mathrm{dB}, 500-\mathrm{Hz}$ pure tone injected into the NIT to illustrate the variability of measured particle displacement as a function of laser synchronization. Here phase is defined as the time delay between the start of one cycle of the acoustic wave and the first laser pulse. The results can be seen in Figure 7, with the field of view of the camera centered approximately $46 \mathrm{~mm}$ from the termination. The initial data point was taken with the first laser pulse located near the peak of the acoustic wave. Each subsequent data point represents an acquisition where the first laser pulse was delayed an additional $1 / 16$ of a wave. Clearly it can be seen that the position of the first laser pulse in reference to the acoustic wave peak is critical towards obtaining accurate displacement information. The correct maximum displacement occurs for only two particular phase angles, one yielding a positive and the other a negative displacement. Although it is tedious to acquire the data, it is important to verify this phase dependency whenever the configuration of the NIT or the sound field is changed so that the correct synchronization phase can be chosen.

Sound Pressure Level Variation: A $500-\mathrm{Hz}$ pure tone was injected into the NIT with the sound pressure level varied from 90 to $132 \mathrm{~dB}$ in steps of $3 \mathrm{~dB}$ to determine the minimum threshold at which acoustic particle displacements could be resolved (one of the fundamental goals of this study). The trigger microphone was used to verify the sound pressure levels in the NIT. Figure 8 shows the results of the measurements plotted on a semi-log scale, with the field of view of the camera in the same position as for Figure 7. As expected, the acoustic particle displacement doubles for every $6 \mathrm{~dB}$ increase in sound pressure level. The graph also indicates a minimum particle displacement detection limit of approximately one micrometer at a sound pressure level of approximately $99 \mathrm{~dB}$. The ability to detect displacements in the micrometer range is a direct result of using the high quality diffraction-limited receiver lens and calcite image shifter described previously, coupled with the ability to resolve 0.1-pixel changes in the spatial autocorrelation peak.

A surprising result was also observed near the upper end of the curve in Figure 8. It was expected that above $120-125 \mathrm{~dB}$, harmonic frequencies and acoustic streaming would begin to become noticeable, causing a deviation from the linear fit shown on the graph. However, no such deviation was seen at sound pressure levels up to $132 \mathrm{~dB}$, the maximum levels tested, although the uncertainty in the measurement did increase slightly from those observed at the mid-range sound pressure levels of approximately $120 \mathrm{~dB}$. 
Centerline Survey of NIT: Figure 9 shows the results of a centerline survey conducted in the NIT for a pure tone frequency of $1000 \mathrm{~Hz}$ at a sound pressure level of $116 \mathrm{~dB}$. The acoustic particle displacement and velocity were measured as a function of distance from the termination, spanning a total range of approximately $50 \mathrm{~mm}$. The field of view of the camera was adjusted to $7.6 \times 6.0 \mathrm{~mm}$, overlapped $50 \%$ between adjacent survey locations. Also shown in Figure 9 is a curve generated using equations (2) - (4), showing the predicted acoustic particle displacement and velocity as a function of distance from the termination. Good agreement is seen between the experimental data and the theory in Figure 9, with all measured data points falling on the theoretical line within the bounds of the error bars.

\section{ERROR ANALYSIS}

A generalized error analysis for these measurements requires that two main error sources be quantified, namely the degree of accuracy with which entrained seed particles in the NIT follow the acoustic field, and the accuracy of individual particle image displacement and velocity measures obtained with the DPIV system. Both of these error sources are described below. Only precision errors in the measurements are addressed - it is assumed that any bias errors are negligible and/or are removed through calibration of the DPIV instrument.

Particle Tracking Fidelity: Numerous theoretical studies of the dynamic tracking fidelity of suspended particles in a fluid medium have been discussed in the literature, with some of the earliest work presented by Soo $^{16}$, Somerscales ${ }^{17}$, Mazumder and $\mathrm{Kirsch}^{18}$, and Walsh. ${ }^{19}$ Many of these investigations concentrated on the impact of particle response on the performance of Laser Doppler Velocimeter (LDV) measurements in turbulent flows; however, these same theoretical studies can be extended to estimate the acoustic particle displacement tracking accuracy for DPIV. A critical quantity determining the response of the particle to the sound field is defined as the ratio of the particle velocity to the gas velocity, defined by Mazumder in reference 18 as the "degree of tracking fidelity" and given by

$$
\mu_{p}=\left(\frac{u_{p}}{u_{g}}\right)=\frac{1}{\sqrt{1+\omega^{2} \tau_{p}^{2}}}
$$

where $\tau_{p}$ is the dynamic relaxation time of the particle in a one-dimensional oscillatory flow field, defined as

$$
\tau_{p}=\frac{\rho_{p} d_{p} C_{c}}{18 \bar{\eta}}
$$

where $\rho_{p}$ is the particle density, $d_{p}$ is the particle diameter, $C_{c}$ is the Cunningham correction factor for molecular slip, and $\bar{\eta}$ is the average fluid viscosity. For a particle suspended in a zero-mean, oscillatory flow, one can assume that the Reynolds number, $R e$, is less than one and that $C_{c}$ is approximately equal to one, implying that particle motion is governed primarily by Stokes' law.

Figure 10 shows $\mu_{p}$ versus frequency of oscillation for hollow silicon dioxide particles with the following characteristics:

\begin{tabular}{|l|l|}
\hline Particle Density & $0.45 \mathrm{grams} / \mathrm{cm}^{3}$ \\
\hline Air Viscosity & $18.1 \mu \mathrm{N}-\mathrm{sec} / \mathrm{m}^{2}$ \\
\hline Particle Diameter & $2.0,2.7,3.0 \mu \mathrm{m}$ \\
\hline Frequency of Oscillation & $0-10 \mathrm{kHz}$ \\
\hline
\end{tabular}

The 2.0- and 3.0- $\mu \mathrm{m}$ particle diameters are shown to provide a bracket for the $2.7-\mu \mathrm{m}$ particle size used for this study. It is immediately evident from Figure 10 that for the frequencies employed (which never exceeded $1 \mathrm{kHz}$ for this study) that the particles follow the acoustic field with greater than $99 \%$ fidelity.

Displacement / Velocity Measure Uncertainty: The literature contains several PIV and DPIV uncertainty investigations. Early work concentrated on both the identification of spurious vectors within PIV-generated velocity fields and on optimization of the instrument to minimize these errors. ${ }^{20-21}$ More recently, Lourenco and Krothapalli ${ }^{22}$, and Huang, Dabiri, and Gharib ${ }^{23}$ have provided techniques for minimizing root-meansquare errors in location estimates of correlation plane peaks. Given that the DPIV system must resolve particle image displacements on the order of $1 \mu \mathrm{m}$, it was desired to build on these previous investigations by performing a simplified precision error propagation analysis.

It can be shown using a Taylor series expansion (See Appendix A) that the precision errors associated with calculation of individual DPIV displacement and velocity vectors are given by 


$$
\begin{aligned}
\sigma_{\mathrm{D}}^{2} \approx & \left(\frac{1}{M}\right)^{2} \sigma_{\mathrm{d}}^{2}+\left(\frac{1}{M}\right)^{2} \sigma_{\mathrm{d}_{s}}^{2}+\left(\frac{\mathrm{d}_{s}-\mathrm{d}}{M^{2}}\right)^{2} \sigma_{M}^{2} \\
& -2\left(\frac{1}{M}\right)^{2} \sigma_{\mathrm{d}} \sigma_{\mathrm{d}_{s}}+2\left(\frac{1}{M}\right)\left(\frac{\mathrm{d}_{\mathrm{s}}-\mathrm{d}}{M^{2}}\right) \sigma_{\mathrm{d}} \sigma_{M} \\
& +2\left(\frac{1}{M}\right)\left(\frac{\mathrm{d}-\mathrm{d}_{s}}{M^{2}}\right) \sigma_{\mathrm{d}_{s}} \sigma_{M}
\end{aligned}
$$

and

$$
\begin{aligned}
\sigma_{\mathrm{V}}^{2} \approx & \left(\frac{1}{M \Delta \tau}\right)^{2} \sigma_{\mathrm{d}}^{2}+\left(\frac{1}{M \Delta \tau}\right)^{2} \sigma_{\mathrm{d}_{s}}^{2}+\left(\frac{\mathrm{d}_{s}-\mathrm{d}}{M^{2} \Delta \tau}\right)^{2} \sigma_{M}^{2} \\
& +\left(\frac{\mathrm{d}_{s}-\mathrm{d}}{M \Delta \tau^{2}}\right)^{2} \sigma_{\Delta \tau}^{2}-2\left(\frac{1}{M \Delta \tau}\right)^{2} \sigma_{\mathrm{d}} \sigma_{\mathrm{d}_{s}} \\
& +2\left(\frac{1}{M \Delta \tau}\right)\left(\frac{\mathrm{d}_{s}-\mathrm{d}}{M^{2} \Delta \tau}\right) \sigma_{\mathrm{d}} \sigma_{M} \\
& +2\left(\frac{1}{M \Delta \tau}\right)\left(\frac{\mathrm{d}-\mathrm{d}_{s}}{M^{2} \Delta \tau}\right) \sigma_{\mathrm{d}_{s}} \sigma_{M}
\end{aligned}
$$

where the $\sigma$ terms represent the precision errors of the variables appearing in equation (6). To compute an upper bound on the precision errors expected for the particle displacements and velocities shown in Figures 7 through 9, the following mean values and standard deviations were assumed:

\begin{tabular}{|l|l|l|}
\hline Variable & Mean & Standard Deviation, $\sigma$ \\
\hline$M$ & 1.0 & 0.05 \\
\hline$\Delta \tau$ & $500 \mu \mathrm{Sec}$ & $5 \mathrm{nSec}$ \\
\hline$d$ & $1-100 \mu \mathrm{m}$ & $0.5 \mu \mathrm{m}$ \\
\hline$d_{s}$ & $180 \mu \mathrm{m}$ & $0.1 \mu \mathrm{m}$ \\
\hline
\end{tabular}

The standard deviations were chosen based on conservative estimates of expected precision errors in the measurement of each variable. For instance, the standard deviation in the measured displacement, $d$, is based on an assumption that the correlation peak can be located to 0.1-pixel accuracy.

Figure 11 shows velocity uncertainties both calculated using equation (10) and obtained experimentally using the data shown in Figure 8. The results indicate that the standard deviations listed in the table above are reasonable estimates, and that the error propagation analysis does indeed provide an upper bound for the velocity precision error. The two measured error points which rise above the error propagation curve in Figure 11 are most likely due to nonlinear acoustic effects introduced into the experiment, rather than being due to DPIV precision errors. The sound pressure levels corresponding to these two points are both greater than $130 \mathrm{~dB}$, most likely generating unwanted harmonics, distortions in the sound field, and acoustic streaming which can bias the acoustic particle displacement and velocity measurements.

\section{CONCLUDING REMARKS}

An investigation of the application of DPIV techniques for the measurement of acoustic particle displacement and velocity in a normal incidence impedance tube zero-mean flow has been performed. The tests have shown that the DPIV system is capable of measuring particle displacements down to approximately $1 \mu \mathrm{m}$ for sound pressure levels as low as $100 \mathrm{~dB}$. At higher sound pressure levels (approaching $132 \mathrm{~dB}$ ) where nonlinear acoustic medium effects were observed, the PIV system was still capable of extracting accurate displacement measurements. A centerline acoustic particle / velocity displacement survey of the NIT agreed well with theoretical predictions. These results indicate a remarkable ability to measure micrometer-range particle displacements in a zeromean flow, and represent the first measurements of this kind obtained with PIV techniques.

Two types of error analyses were performed as part of the study. A particle tracking fidelity analysis revealed that the $2.7-\mu \mathrm{m}$ hollow silicon dioxide seed particles employed for the study followed the acoustic field inside the NIT with acceptable accuracy, with the degree of tracking fidelity exceeding $99 \%$. Also, a comparison of measured and theoretical precision errors showed acceptable agreement, with an upper uncertainty bound established based on a propagation of errors analysis.

In summary, the results of this study are encouraging. However, it must be noted that more work is required to determine if this technique is applicable when measurements of a sound field in a non-zero mean flow are required. This represents a more real-world condition for testing acoustic nacelle liners, the ultimate goal of such experiments. Measurements of acoustic particle displacement and velocity in these more complex configurations are the subject of current research.

\section{ACKNOWLEDGMENTS}

The authors wish to gratefully acknowledge the assistance of James L. Blackshire, Vigyan, Inc., in development of DPIV data processing and validation codes and in the analysis of several of the examples data sets presented here. 


\section{REFERENCES}

1. Pierce, Allan D., "Acoustics - An Introduction to Its Physical Principles and Applications", Acoustic Society of America, Woodbury, New York, 1989.

2. Jones, M. G., and Stiede, P. E., "Comparison of Methods for Determining Specific Acoustic Impedance", Journal of the Acoustic Society of America, Volume 101, Number 5, pp. 2694-2704, 1997.

3. Sharpe, J. P., and Greated, C. A., "The Measurement of Periodic Acoustic Fields Using Photon Correlation Spectroscopy", Journal of Physics D: Applied Physics, Volume 20, pp. 418423, 1987.

4. Sharpe, J. P., Greated, C. A., and Campbell, D. M., "The Measurement of Complex Acoustic Impedance Using Photon Correlation Spectroscopy", Acoustica, Volume 66, pp. 266$289,1988$.

5. Sharpe, J. P., Greated, C. A., Gray, C., and Campbell, D. M., "The Measurement of Acoustic Streaming using Particle Image Velocimetry", Acoustica, Volume 68, pp. 168-172, 1988.

6. Arroyo, M. P., and Greated, C. A., "A Three Dimensional Particle Image Velocimetry System and Its Application to the Measurement of Acoustic Streaming", Flow Visualization and Image Analysis, F. T. M. Nieuwstadt (ed.), pp. 167$188,1993$.

7. Hann, D. B., and Greated, C. A., "The Measurement of Flow Velocity and Acoustic Particle Velocity Using Particle-Image Velocimetry", Measurement Science and Technology, Volume 8, pp. 1517-1522, 1997.

8. Lighthill, James, "Acoustic Streaming", Journal of Sound and Vibration, Volume 61, Number 3, pp. 391-418, 1978.

9. Humphreys, W. M., Jr., Bartram, S. M., and Blackshire, J. L., "A Survey of Particle Image Velocimetry Applications in Langley Aerospace Facilities", AIAA Paper 93-0411, 31 $1^{\text {st }}$ Aerospace Sciences Meeting, Reno, NV, 1993.
10. Adrian, R. J., "Image Shifting Technique to Resolve Directional Ambiguity in Double-Pulsed Velocimetry", Applied Optics, Volume 25, pp. 3855-3858, 1986.

11. Landreth, C. C., and Adrian, R.J., "Electrooptical Image Shifting for Particle Image Velocimetry", Applied Optics, Volume 27, pp. 4216-4220, 1988.

12. Humphreys, W. M., Jr., Bartram, S. M., and Clark, I.O., "Velocity Field Measurements in a Chemical Vapor Deposition Reactor Using Digital Particle Image Velocimetry", Proceedings of the ASME Symposium on Laser Anemometry and Experimental and Numerical Flow Visualization, San Diego, CA, 1996.

13. Blackshire, J. L., "Analysis of Particle Image Velocimetry (PIV) Data for Acoustic Velocity Measurements", NASA Contractor Report 201664, 1997.

14. Adrian, R. J., "PIV Processing Technique: Image Plane and Fourier Plane", von Karman Institute for Fluid Dynamics Lecture Series 1988-06: Particle Image Velocimetry, Brussels, Belgium, 1988.

15. Blackshire, J. L., "PIV Data Validation Software Package", NASA Contractor Report 201701, 1997.

16. Soo, S. L., Fluid Dynamics of Multiphase Systems, Blaesdell Publishing Company, Waltham, Massachussetts, 1967.

17. Somerscales, E. F. C., "The Dynamic Characteristics of Flow Tracing Particles", Proceedings of the $2^{\text {nd }}$ International Workshop on Laser Velocimetry, Purdue University, pp. 216231, 1974.

18. Mazumder, M. K., and Kirsch, K. J., "Flow Tracing Fidelity of Scattering Aerosol in Laser Doppler Velocimetry", Applied Optics, Volume 14, pp. 894-901, 1975.

19. Walsh, M. J., "Influence of Particle Drag Coefficient on Particle Motion in High-Speed Flow with Typical Laser Velocimeter Applications", NASA Technical Note D-8120, 1976.

20. Keane, R. D., and Adrian, R. J., "Optimization of Particle Image Velocimeters, Part 1: Double 
Pulsed Systems", Measurement Science and Technology, Volume 1, pp. 1202-1215, 1990.

21. Host-Madsen, A., and McCluskey, D. R., "On the Accuracy and Reliability of PIV Measurements", Seventh International Symposium on Applications of Laser Anemometry to Fluid Mechanics, Lisbon, Portugal, paper 26.4, 1994.

22. Lourenco, L., and Krothapalli, A., "On the Accuracy of Velocity and Vorticity Measurements with PIV", Experiments in Fluids, Volume 18, pp. 421-428, 1995.

23. Huang, H., Dabiri, D., and Gharib, M., "On Errors of Digital Particle Image Velocimetry", Measurement Science and Technology, Volume 8, pp. 1427-1440, 1997.

24. Measurement Uncertainty, ANSI / ASME Performance Test Codes (PTC) 19.1-1985, Part 1, American Society of Mechanical Engineers, Fairfield, New Jersey, 1985.

\section{APPENDIX A - DERIVATION OF PRECISION ERRORS FOR DPIV MEASUREMENTS}

As seen in equation (6), a DPIV velocity measurement relies on only four arguments, i.e., $\overrightarrow{\mathrm{V}}=f\left(\overrightarrow{\mathrm{d}}, \overrightarrow{\mathrm{d}}_{s}, M, \Delta \tau\right)$.

A classical precision error propagation can be performed by applying a Taylor series expansion to the variables represented in equation (6). Using notation similar to that employed in reference 24 , and dropping vector symbol notation for convenience, one can write the Taylor expansion as follows

$$
\begin{aligned}
\mathrm{V}= & \mu_{\mathrm{V}}+\frac{\partial \mathrm{V}}{\partial \mathrm{d}}\left(\mathrm{d}-\mu_{\mathrm{d}}\right)+\frac{\partial \mathrm{V}}{\partial \mathrm{d}_{s}}\left(\mathrm{~d}_{s}-\mu_{\mathrm{d}_{s}}\right) \\
& +\frac{\partial \mathrm{V}}{\partial \mathrm{M}}\left(M-\mu_{\mathrm{M}}\right)+\frac{\partial \mathrm{V}}{\partial \Delta \tau}\left(\Delta \tau-\mu_{\Delta \tau}\right)+R_{2}
\end{aligned}
$$

Assuming $R_{2}$ to be small, equation (A.1) can be rewritten as

$$
\begin{aligned}
\mathrm{V}-\mu_{\mathrm{V}} & =\frac{\partial \mathrm{V}}{\partial \mathrm{d}}\left(\mathrm{d}-\mu_{\mathrm{d}}\right)+\frac{\partial \mathrm{V}}{\partial \mathrm{d}_{s}}\left(\mathrm{~d}_{s}-\mu_{\mathrm{d}_{s}}\right) \\
& +\frac{\partial \mathrm{V}}{\partial \mathrm{M}}\left(M-\mu_{\mathrm{M}}\right)+\frac{\partial \mathrm{V}}{\partial \Delta \tau}\left(\Delta \tau-\mu_{\Delta \tau}\right)
\end{aligned}
$$

The variation in the velocity estimate can be defined in terms of the probability density function of $\mathrm{V}$ as

$$
\sigma_{\mathrm{V}}^{2}=\left(\mathrm{V}-\mu_{\mathrm{V}}\right)^{2} P_{\mathrm{V}} d \mathrm{~V}
$$

Substituting equation (A.2) into (A.3) and integrating, one obtains

$$
\begin{aligned}
\sigma_{\mathrm{V}}^{2} \approx & \left(\frac{\partial \mathrm{V}}{\partial \mathrm{d}}\right)^{2} \sigma_{\mathrm{d}}^{2}+\left(\frac{\partial \mathrm{V}}{\partial \mathrm{d}_{s}}\right)^{2} \sigma_{\mathrm{d}_{s}}^{2} \\
& +\left(\frac{\partial \mathrm{V}}{\partial M}\right)^{2} \sigma_{M}^{2}+\left(\frac{\partial \mathrm{V}}{\partial \tau}\right)^{2} \sigma_{\Delta \tau}^{2} \\
& +2 \frac{\partial \mathrm{V}}{\partial \mathrm{d}} \frac{\partial \mathrm{V}}{\partial \mathrm{d}_{s}} \rho_{\mathrm{d}_{\mathrm{d}, \mathrm{d}_{s}}} \sigma_{\mathrm{d}} \sigma_{\mathrm{d}_{s}} \\
& +2 \frac{\partial \mathrm{V}}{\partial \mathrm{d}} \frac{\partial \mathrm{V}}{\partial M} \rho_{\mathrm{d}, M} \sigma_{\mathrm{d}} \sigma_{M} \\
& +2 \frac{\partial \mathrm{V}}{\partial \mathrm{d}} \frac{\partial \mathrm{V}}{\partial \Delta \tau} \rho_{\mathrm{d}, \Delta \tau} \sigma_{\mathrm{d}} \sigma_{\Delta \tau} \\
& +2 \frac{\partial \mathrm{V}}{\partial \mathrm{d}_{s}} \frac{\partial \mathrm{V}}{\partial M} \rho_{\mathrm{d}_{s}, M} \sigma_{\mathrm{d}_{s}} \sigma_{M} \\
& +2 \frac{\partial \mathrm{V}}{\partial \mathrm{d}_{s}} \frac{\partial \mathrm{V}}{\partial \Delta \tau} \rho_{\mathrm{d}_{s}, \Delta \tau} \sigma_{\mathrm{d}_{s}} \sigma_{\Delta \tau} \\
& +2 \frac{\partial \mathrm{V}}{\partial M} \frac{\partial \mathrm{V}}{\partial \Delta \tau} \rho_{M, \Delta \tau} \sigma_{M} \sigma_{\Delta \tau}
\end{aligned}
$$

where the $\sigma$ terms represent the precision errors of the individual variables appearing in equation (6), and the $\rho$ terms represent the correlation coefficients between pairs of variables. Some simplifying assumptions can be made at this point. For instance, it is assumed that $\Delta \tau$ is independent with respect to the other variables; therefore, $\rho_{d, \Delta \tau}=\rho_{d_{s}, \Delta \tau}=\rho_{M, \Delta \tau}=0$. However, this same assumption cannot be made regarding the other correlation coefficients appearing in (A.4), since errors in the measurement of magnification and image shift manifest themselves as errors in measurement of the particle image displacement. For simplicity, to obtain an accurate upper bound estimate of the velocity precision error, let us assume that $\rho_{d, d_{s}}=\rho_{d_{s} M}=\rho_{d_{s}, M}=1$. Then, equation (A.4) simplifies to

$$
\begin{aligned}
\sigma_{\mathrm{V}}^{2} & \approx\left(\frac{\partial \mathrm{V}}{\partial \mathrm{d}}\right)^{2} \sigma_{\mathrm{d}}^{2}+\left(\frac{\partial \mathrm{V}}{\partial \mathrm{d}_{s}}\right)^{2} \sigma_{\mathrm{d}_{s}}^{2} \\
& +\left(\frac{\partial \mathrm{V}}{\partial M}\right)^{2} \sigma_{M}^{2}+\left(\frac{\partial \mathrm{V}}{\partial \Delta \tau}\right)^{2} \sigma_{\Delta \tau}^{2} \\
& +2 \frac{\partial \mathrm{V}}{\partial \mathrm{d}} \frac{\partial \mathrm{V}}{\partial \mathrm{d}_{s}} \sigma_{\mathrm{d}} \sigma_{\mathrm{d}_{s}} \\
& +2 \frac{\partial \mathrm{V}}{\partial \mathrm{d}} \frac{\partial \mathrm{V}}{\partial M} \sigma_{\mathrm{d}} \sigma_{M} \\
& +2 \frac{\partial \mathrm{V}}{\partial \mathrm{d}_{s}} \frac{\partial \mathrm{V}}{\partial M} \sigma_{\mathrm{d}_{s}} \sigma_{M}
\end{aligned}
$$

To reduce this expression to a useable form, compute the partial differentials appearing in (A.5) using equation (6) as 


$$
\begin{aligned}
& \frac{\partial \mathrm{V}}{\partial \mathrm{d}}=\frac{1}{M \Delta \tau} \\
& \frac{\partial \mathrm{V}}{\partial \mathrm{d}_{s}}=\frac{-1}{M \Delta \tau} \\
& \frac{\partial \mathrm{V}}{\partial M}=\frac{\mathrm{d}_{s}-\mathrm{d}}{M^{2} \Delta \tau} \\
& \frac{\partial \mathrm{V}}{\partial \Delta \tau}=\frac{\mathrm{d}_{s}-\mathrm{d}}{M \Delta \tau^{2}}
\end{aligned}
$$

Substituting the above into (A.5) and simplifying, one obtains

$$
\begin{aligned}
\sigma_{\mathrm{V}}^{2} \approx & \left(\frac{1}{M \Delta \tau}\right)^{2} \sigma_{\mathrm{d}}^{2}+\left(\frac{1}{M \Delta \tau}\right)^{2} \sigma_{\mathrm{d}_{s}}^{2}+\left(\frac{d_{s}-\mathrm{d}}{M^{2} \Delta \tau}\right)^{2} \sigma_{M}^{2} \\
& +\left(\frac{d_{s}-\mathrm{d}}{M \Delta \tau^{2}}\right)^{2} \sigma_{\Delta \tau}^{2}-2\left(\frac{1}{M \Delta \tau}\right)^{2} \sigma_{\mathrm{d}} \sigma_{\mathrm{d}_{s}} \\
& +2\left(\frac{1}{M \Delta \tau}\right)\left(\frac{d_{s}-\mathrm{d}}{M^{2} \Delta \tau}\right) \sigma_{\mathrm{d}} \sigma_{M} \\
& +2\left(\frac{1}{M \Delta \tau}\right)\left(\frac{d-d_{s}}{M^{2} \Delta \tau}\right) \sigma_{d_{s}} \sigma_{M}
\end{aligned}
$$

To obtain the particle image displacement precision error, set $\Delta \tau=1$ and $\sigma_{\Delta \tau}=0$ in (A.7) to obtain

$$
\begin{aligned}
\sigma_{\mathrm{D}}^{2} \approx & \left(\frac{1}{M}\right)^{2} \sigma_{\mathrm{d}}^{2}+\left(\frac{1}{M}\right)^{2} \sigma_{\mathrm{d}_{s}}^{2}+\left(\frac{\mathrm{d}_{s}-\mathrm{d}}{M^{2}}\right)^{2} \sigma_{M}^{2} \\
& -2\left(\frac{1}{M}\right)^{2} \sigma_{\mathrm{d}} \sigma_{\mathrm{d}_{s}}+2\left(\frac{1}{M}\right)\left(\frac{\mathrm{d}_{s}-\mathrm{d}}{M^{2}}\right) \sigma_{\mathrm{d}} \sigma_{M} \\
& +2\left(\frac{1}{M}\right)\left(\frac{\mathrm{d}-\mathrm{d}_{s}}{M^{2}}\right) \sigma_{\mathrm{d}_{s}} \sigma_{M}
\end{aligned}
$$




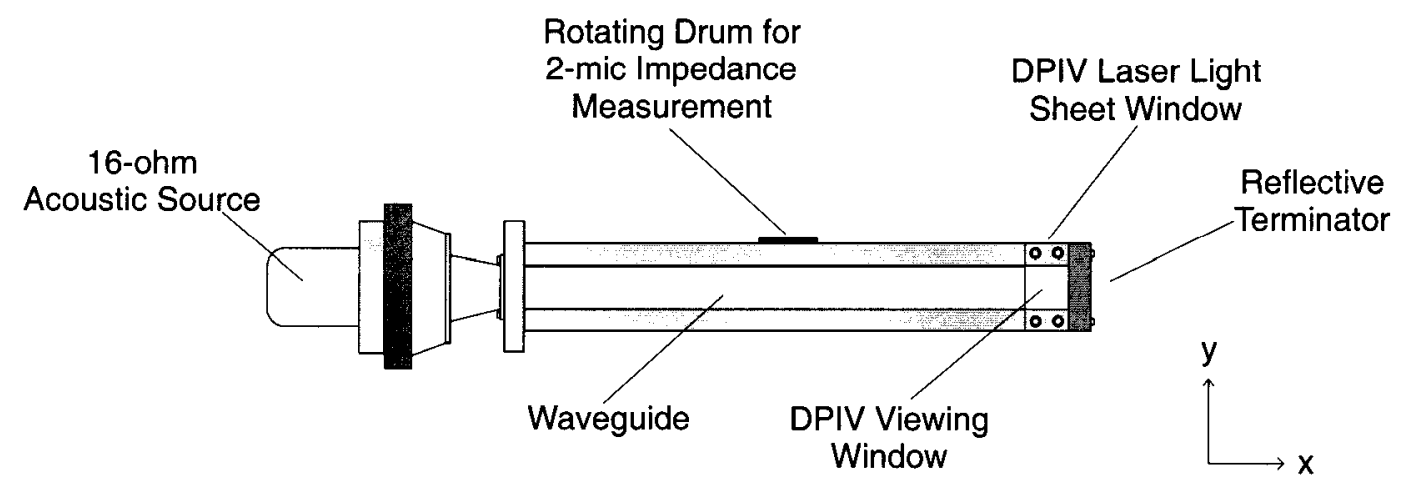

Figure 1. Schematic of Normal Incidence Impedance Tube (NIT).

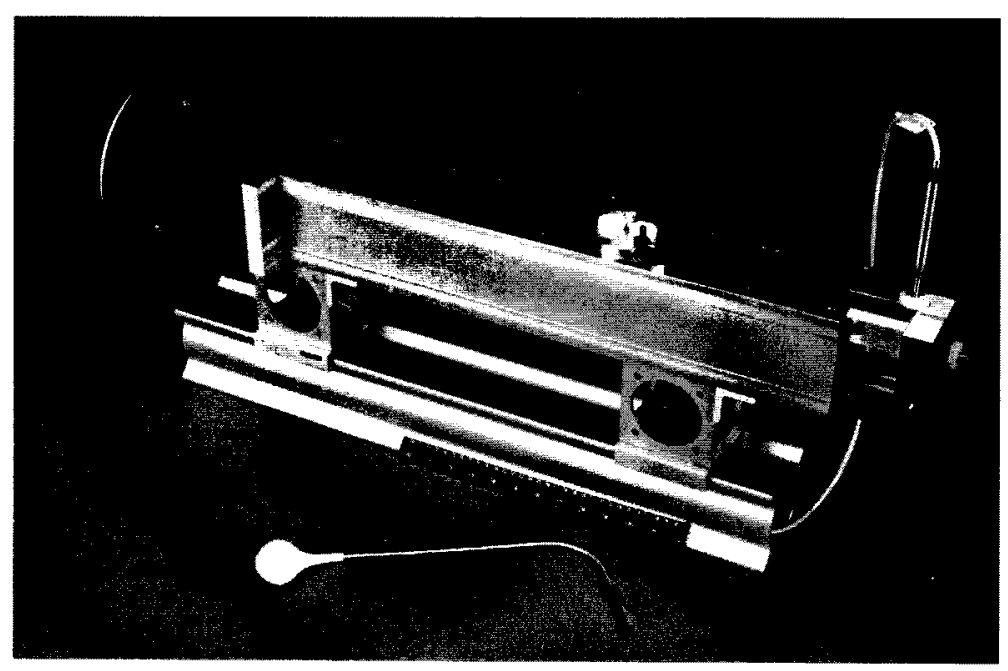

Figure 2. Photo of NIT.

\section{Beam-Combining Optics}

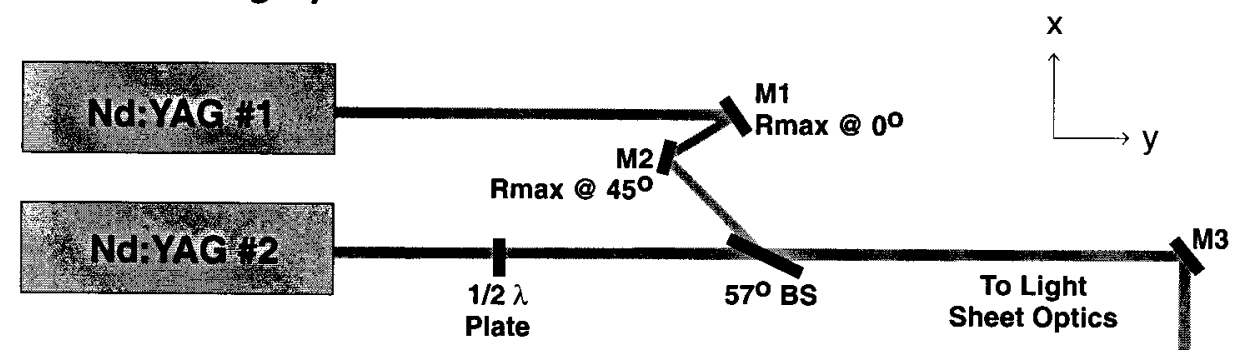

Sheet Forming Optics

Light Sheet

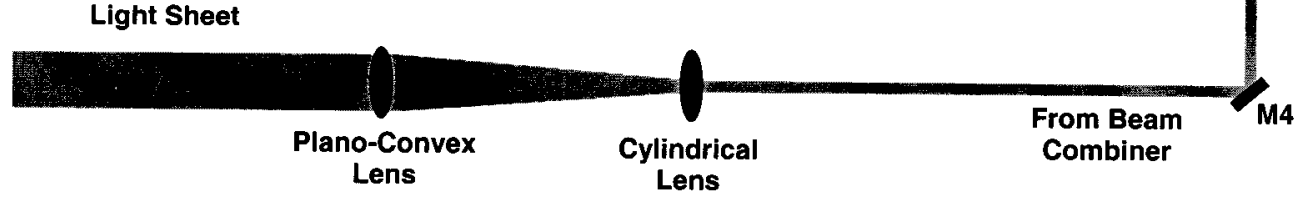

Figure 3. DPIV Laser Optics System. 


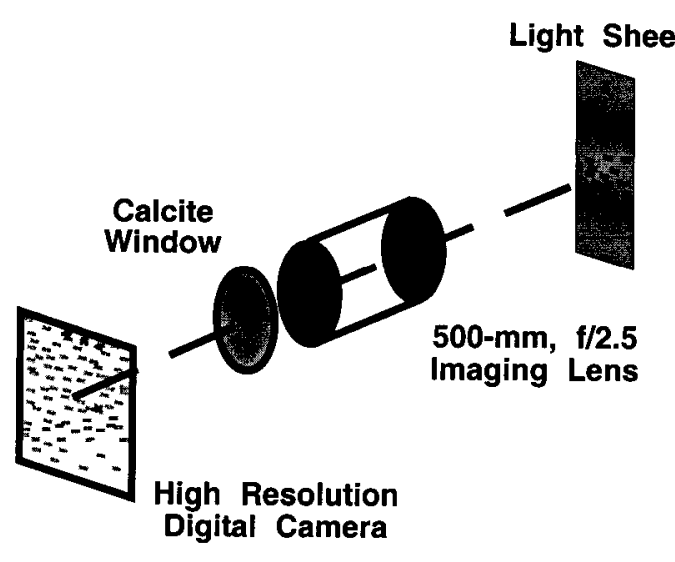

Figure 4. DPIV Receiver Schematic.

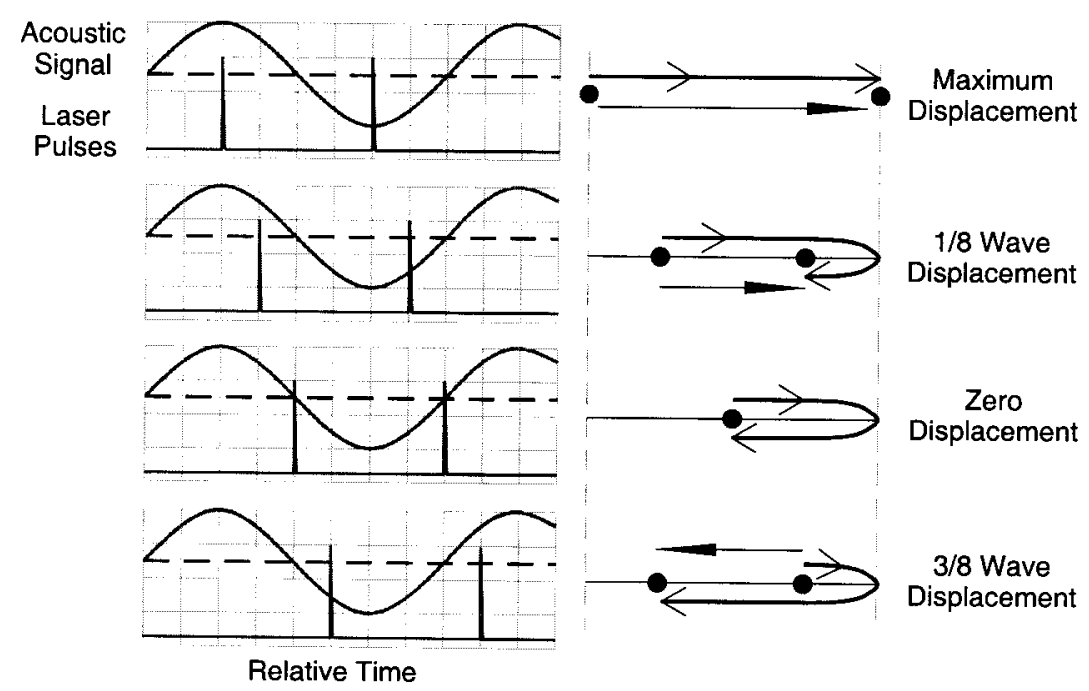

Figure 5. Measured Apparent Particle Displacements as a Function of Phase Synchronization.

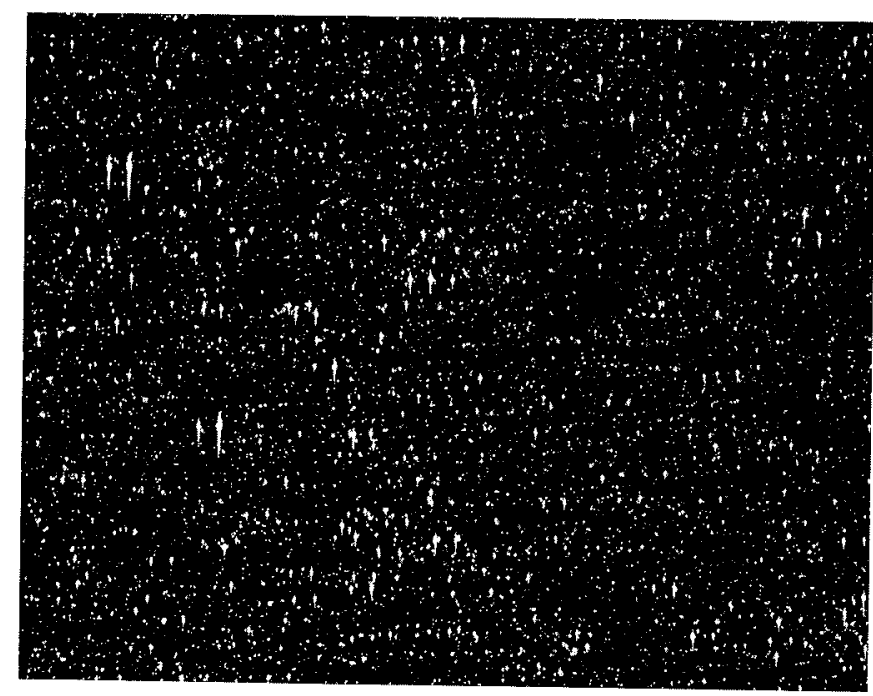

Figure 6. Sample DPIV Image Obtained in NIT. 


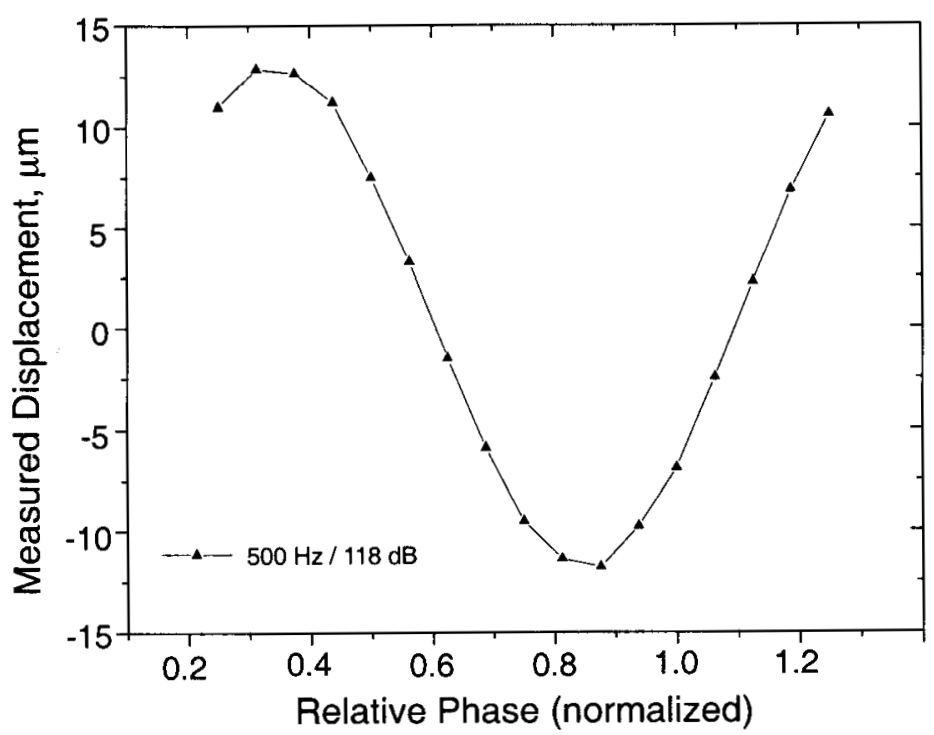

Figure 7. Experimental Verification of Effect Illustrated in Figure 5.

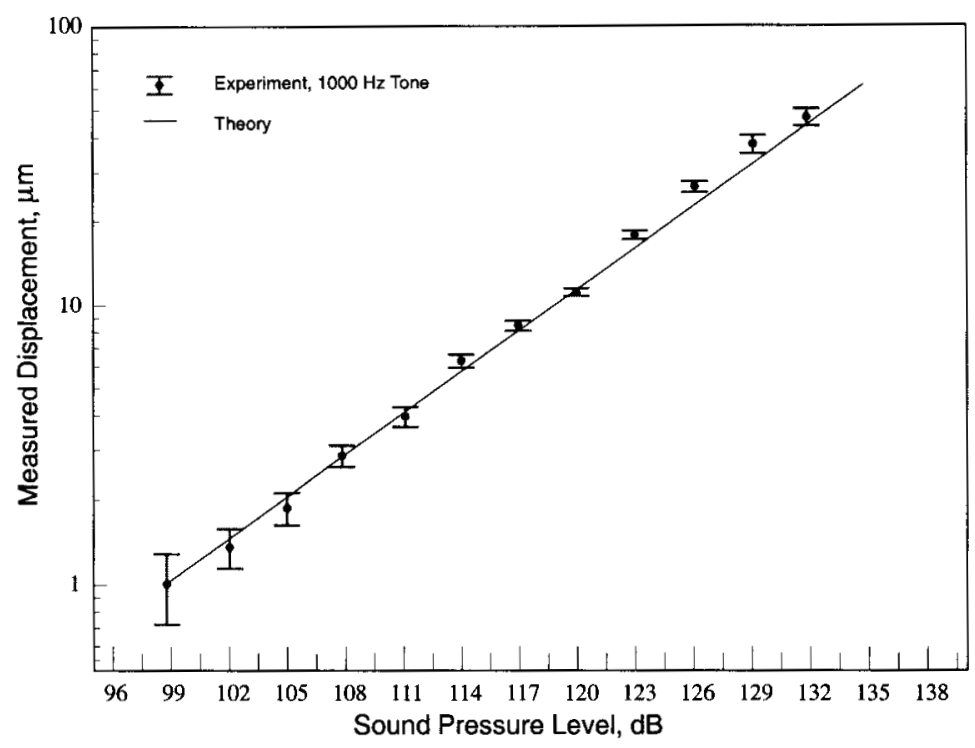

Figure 8. Variation in Acoustic Displacement as Function of Sound Pressure Level.

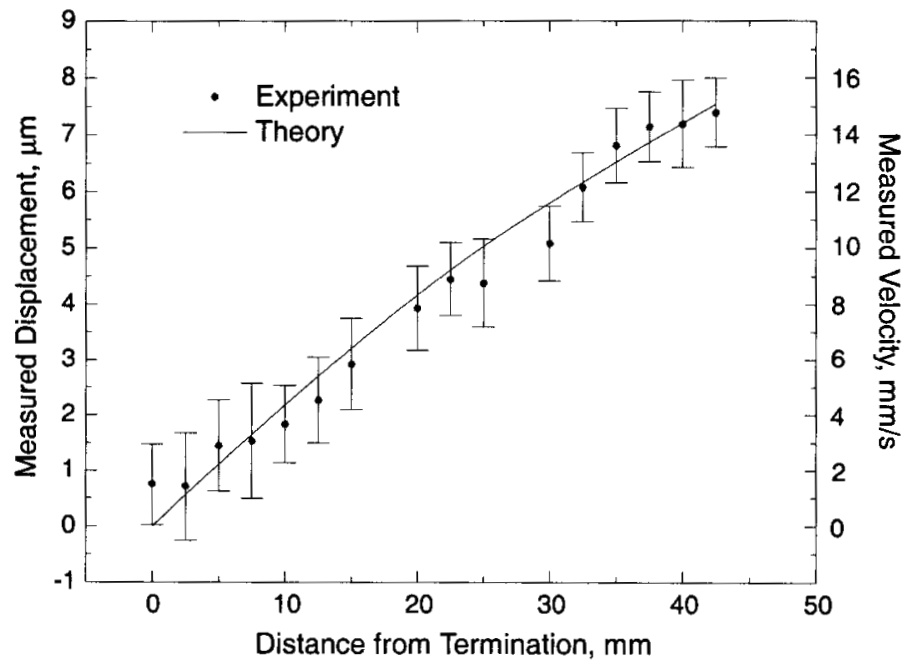

Figure 9. Centerline Survey Showing Acoustic Particle Displacement and Velocity as Function of Distance from NIT Termination. 


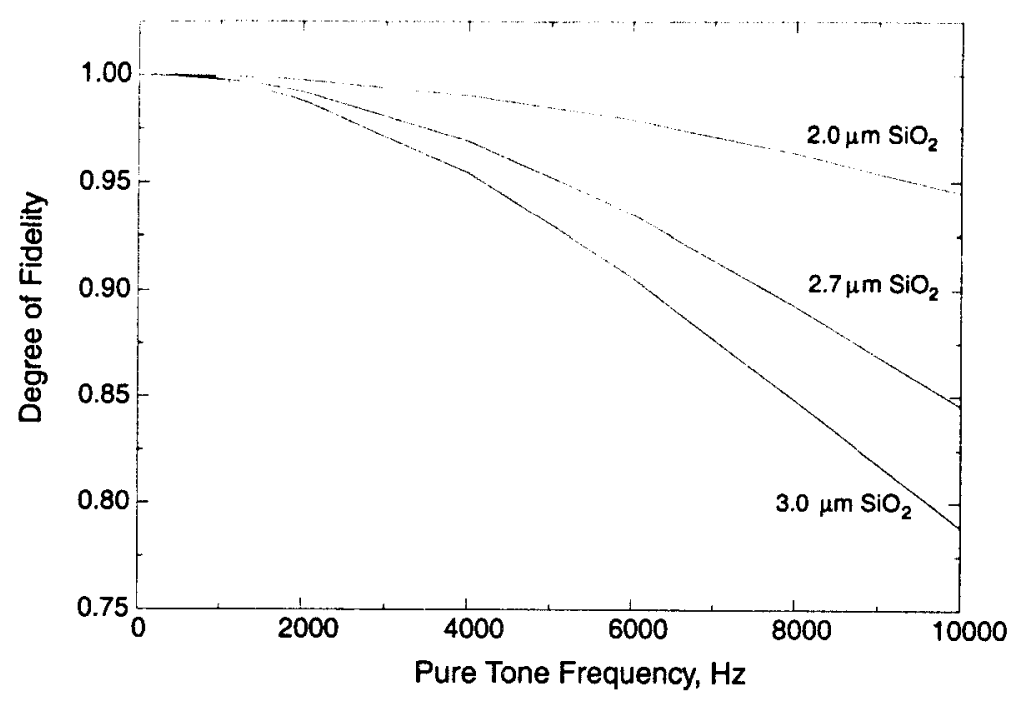

Figure 10. Particle Tracking Fidelity as Function of Acoustic Frequency and Particle Size.

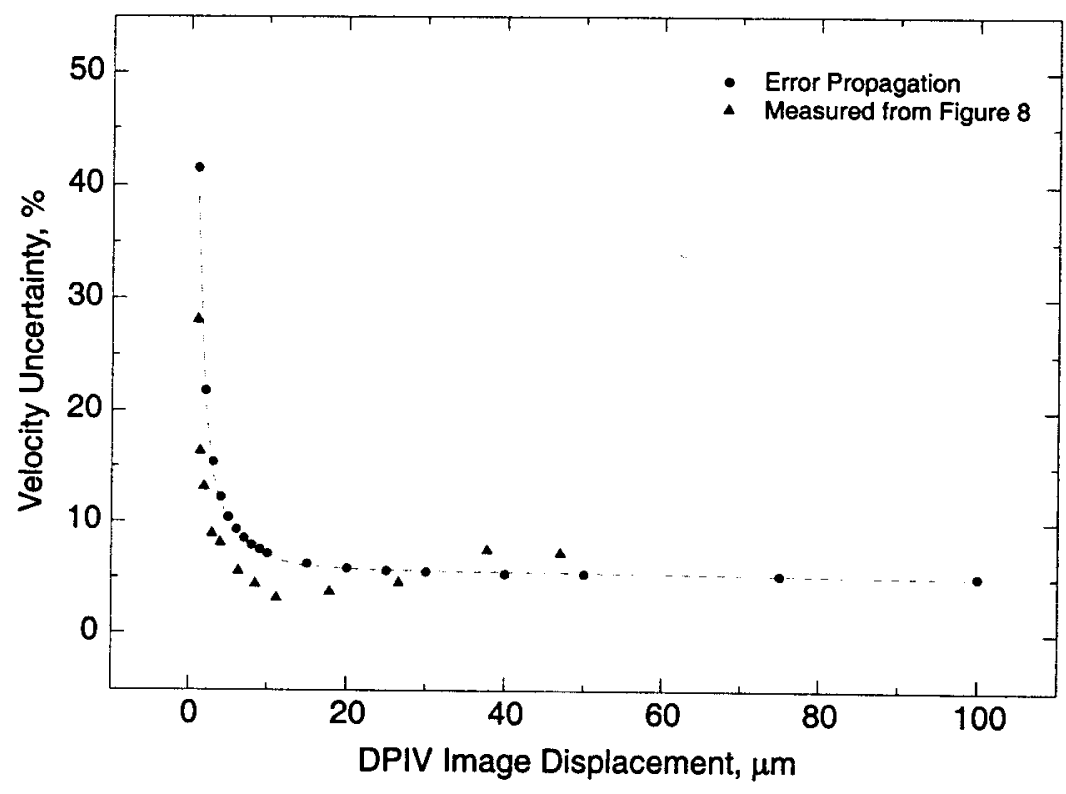

Figure 11. DPIV Velocity Precision Errors. 